autoimmunity for several years prior to the onset of clinical disease. Measuring anti-nuclear antibodies (ANA) is a common test for humoral autoimmunity. It is sensitive $(95+\%)$ for SLE, but not specific as up to $25 \%$ of healthy controls (HC) will have a measurable ANA. While very few of the ANA + HC will develop SLE, they represent a group at higher risk of the disease. Understanding the risks for ANA positivity provides essential knowledge about the development of SLE.

Methods Serum and DNA were collected from 2903 healthy individuals with no personal history of autoimmunity. Antinuclear antibodies were detected using Inova QuantaLite ELISA. Sera from subset $(n=724)$ individuals (ANA- HC, ANA+ $\mathrm{HC}$, and SLE) were assayed by protein microarray quantifying IgM and IgG responses to 89 previously known human autoantigens. A nested cohort consisting of all the ANA + Caucasian individuals and age/gender matched ANAcontrols were genotyped using the ImmunoChipv.1 SNP array. Results In HC, 16\% had moderate and 10\% had high levels of IgG ANA. Autoantigen microarray data showed that ANA+ $\mathrm{HC}$ had a high prevalence of antibodies to non-nuclear and cytoplasmic antigens while subjects with SLE predictably produced antibodies to a variety of nuclear antigens as well. A quantitative genetic association test with ANA identified the locus c11orf30 or EMSY, associated with high ANA phenotype in the healthy population (see figure 1). This locus codes for a negative transcriptional regulator. A haplotype comprised of many potentially regulatory polymorphisms at EMSY contributed to strong risk for ANA in healthy individuals $[(p=3.83 \mathrm{E}$ 04, OR=2.6)]. eQTL data suggests that the ANA-associated EMSY haplotype leads to reduced expression of EMSY protein in human macrophages and EBV cell lines. Autoantibody profiles of serum samples from healthy individuals with the EMSY risk genotype exhibited high titers of anti-IgG and IgA antibodies targeted to multiple autoantigens as well as food and environmental allergens.

Conclusions EMSY, a locus previously linked with atopy, psoriasis and inflammatory bowel disease was associated with ANA in healthy individuals. The EMSY protein is a BRCA2-associated transcriptional repressor. Individuals with risk haplotypes in EMSY make a wide variety of disease-associated antibodies, suggesting an early common pathway for autoimmune and allergic conditions.

\section{GG-08 IMMUNE REPERTOIRE AND GENETIC RISK ALLELES IN HEALTHY PEDIATRIC POPULATIONS WITH AUTOIMMUNE INDICATORS}

${ }^{1}$ Prithvi Raj* ${ }^{1}$ Patricia Pichilingue-Reto, ${ }^{1}$ Igor Dozmorov, ${ }^{1}$ Ran Song, ${ }^{1}$ Chaoying Liang, ${ }^{1}$ Carlos Arana, ${ }^{2}$ Brandi Cantarel, 'Daehwan Kim, 'Bo Zhang, 'JinChun Zhou, 'Erika Molina, ${ }^{3}$ Peter K Gregersen, ${ }^{4}$ Judith A James, ${ }^{5}$ David R Karp, ${ }^{6} \mathrm{M}$ Teresa de la Morena, ${ }^{1}$ QuanZhen Li, ${ }^{6}$ Nicolai SC van Oers, ${ }^{1}$ Edward $\mathrm{K}$ Wakeland. 'Department of Immunology, University of Texas Southwestern Medical Center, Dallas, TX USA; ${ }^{2}$ Department of Bioinformatics, University of Texas Southwestern Medical Center, Dallas, TX USA; ${ }^{3}$ Feinstein Institute for Medical Research, Manhasset, New York USA; ${ }^{4}$ Oklahoma Medical Research Foundation, OKlahoma City, OK USA; ${ }^{5}$ Rheumatic Diseases Division, University of Texas Southwestern Medical Center, Dallas, TX USA; ${ }^{6}$ Department of Pediatrics, University of Texas Southwestern Medical Center, Dallas, TX USA

\subsection{6/lupus-2018-Ism.95}

Background The antibody specificities of an infant progressively form in response to infections, environmental exposures, and vaccinations. While many adults develop antibodies to self-antigens, it remains unknown if these are present in infants and toddlers.
Methods Serum, peripheral blood, and clinical data have been collected from 102 healthy children (1-2 year of age). ANA titers were measured by QuantiaLite ELISA (Inova) and reactivity to 125 diverse autoantigens tested by autoantibody array. Targeted sequencing was performed in 60 children to capture HLA alleles and potentially pathogenic genetic variations in 100 plus loci that are implicated in various autoimmune, rheumatic and immune system related diseases. Sequencing libraries were made using KAPA Biosystems kits. Custom target oligos were synthesized from Nimblegen. Deep sequencing was performed using Illumina HiSeq 4000 platform. Sequencing reads were aligned to reference genome and variants were called using GATK Pipeline. Secondary data analysis is done using Metlab, GraphPad Prism, Haploview and Golden Helix programs.

Results Approximately 28\% of very young children have moderate to high-titer autoantibodies, similar to adults, however, no female gender bias was observed. Significant differences between the child and adult immune repertoires were seen. Some samples demonstrated strong signatures of non-nuclear antigens reactivities. Interestingly, the ANA positive group of children exhibited significantly high titers of anti-alpha fodrin $\operatorname{IgG}(p=0.01)$, an autoantibody reported in juvenile Sjogren's syndrome. Analysis of sequencing data identified regulatory polymorphisms in HLA class II and III regions that were associated with ANA positivity. About $8 \%$ of the ANA positive children also carried autoimmune disease associated HLA-DRDQ alleles. HLA alleles and regulatory haplotypes were analyzed in relation to various clinical features in children. Serum C4 level measured in subset of ANA positive children identified few with reduced expression.

Conclusions While the immune repertoire of very young children is typically thought of as naïve and self-tolerant, a significant fraction of very young children makes autoantibodies as detected by commercial ANA ELISA and autoantigen arrays. This supports the conclusion that ANA and other autoantibodies are consequences of general body development and immune upregulation and not markers of pathology. One of the major genetic determinants of 'pre-clinical' autoimmunity as measured by ANA is the human major histocompatibility locus, HLA. Within HLA, several 'endophenotypes' emerge, including expression of a complement protein involved in immune complex removal, as well as multiple proteins associated with antigen presentation, including MHC class II. These findings support the idea that there is quantifiable genetic risk for the development of autoimmunity that can be measured in very young individuals.

\section{GG-09 A ROLE FOR EBNA2 IN MECHANISMS THAT ARE RESPONSIBLE FOR LUPUS AND OTHER AUTOIMMUNE DISEASES}

1,2,3,4,5]B Harley*, ${ }^{1} \mathrm{X}$ Chen, ${ }^{1} \mathrm{M}$ Pujato, ${ }^{1} \mathrm{D}$ Miller, ${ }^{1} \mathrm{~A}$ Maddox, ${ }^{1} \mathrm{C}$ Forney, ${ }^{1} \mathrm{AF}$ Magnusen, ${ }^{1} \mathrm{~A}$ Lynch, ${ }^{6} \mathrm{~K}$ Chetal, ${ }^{7} \mathrm{M}$ Yukawa, ${ }^{4,7,8} \mathrm{~A}$ Barski, ${ }^{4,6} \mathrm{~N}$ Salomonis, ${ }^{1,2,4,5} \mathrm{KM}$ Kaufman, 1,4 LC Kottyan, ${ }^{1,3,4,6}$ MT Weirauch. ${ }^{1}$ Center for Autoimmune Genomics and Etiology (CAGE); ${ }^{2}$ Division of Immunobiology; ${ }^{3}$ Division of Developmental Biology, Cincinnati Children's Hospital Medical Center, Cincinnati, Ohio; ${ }^{4}$ Department of Pediatrics, University of Cincinnati, Cincinnati, Ohio; ${ }^{5}$ US Department of Veterans Affairs Medical Center, Cincinnati, Ohio; ${ }^{6}$ Division of Biomedical Informatics; ${ }^{7}$ Division of Allergy and Immunology; ${ }^{8}$ Division of Human Genetics, Cincinnati Children's Hospital Medical Center, Cincinnati, Ohio, USA

\subsection{6/lupus-2018-Ism.96}

Background Explaining the genetics of many diseases is challenging because most associations localize to incompletely understood regulatory regions. 